\title{
New, organic, volume superconductor at ambient pressure
}

\author{
H. Schwenk, C. P. Heidmann, F. Gross, E. Hess, and K. Andres \\ Walther-Meissner-Institut für Tieftemperaturforschung, D-8046 Garching, \\ West Germany \\ D. Schweitzer \\ Abteilung für Molekularphysik, Max-Planck-Institut für Medizinische Forschung, \\ D-6900 Heidelberg, West Germany \\ H. J. Keller \\ Institut für Anorganische Chemie, Universität Heidelberg, \\ D-6900 Heidelberg, West Germany
}

(Received 7 August 1984)

\begin{abstract}
We have observed volume superconductivity in $\beta$-(BEDT-TTF) ${ }_{2} I_{3}$ ( $\beta$-bis-ethylenedithiolo-tetrathiafulvalene-triiodide) at ambient pressure with a diamagnetic transition temperature of $1.03 \pm 0.02 \mathrm{~K}$.
\end{abstract}

Recently Yagubskii et al. ${ }^{1}$ reported a superconducting (SC) transition in the organic conductor $\beta$-(BEDT-TTF $)_{2} I_{3}$ at ambient pressure, with a transition temperature of 1.45 $K$. In the similar compound (BEDT-TTF) ${ }_{2} \mathrm{ReO}_{4}$, Parkin et al. ${ }^{2}$ had reported on a superconducting transition under pressure $(4 \mathrm{kbar})$ with a transition temperature of $2 \mathrm{~K}$. Together with the Bechgaard salt (TMTSF) ${ }_{2} \mathrm{FSO}_{3}$, which was reported to superconduct below $2.3 \mathrm{~K}$ under a pressure of 6 $\mathrm{kbar},{ }^{3,4}$ these are the highest resistively observed superconducting transitions in organic conductors so far. Recent investigations by us on the $\mathrm{FSO}_{3}$ salt $^{5}$ showed, however, that superconductivity in this crystal is not a volume property: When measured inductively by means of diamagnetic screening currents or by means of the Meissner effect (magnetic flux expulsion on cooling below $T_{c}$ ), we find that less than $2 \%$ of the crystal volume is superconducting and that the diamagnetic transition temperature is $1.5 \mathrm{~K}$. Thus, transitions to small or zero resistivity do not yet prove volume superconductivity.

Our (BEDT-TTF) ${ }_{2} I_{3}$ salt was prepared by electrochemical methods. $^{6,7}$ Plateletlike crystals were identified as the $\alpha$ phase. An investigation of its properties as a function of pressure revealed that the metal-insulator transition at 135 $\mathrm{K}$ could be practically suppressed above $15 \mathrm{kbar}$. Semimetallic behavior was then observed in the helium temperature range with no superconductivity down to $0.1 \mathrm{~K}^{8}$

Recently, Yagubskii et al. reported the synthesis of several other modifications of this salt,,${ }^{9,10}$ some of which showed resistively observed superconducting transitions below 1.6 and $2.5 \mathrm{~K}$. In our preparation of $\alpha$-(BEDTTTF) ${ }_{2} I_{3}$ using tetrahydrofurane as a solvent, ${ }^{6,7}$ we always noted the simultaneous formation of canted rhombohedrons of the $\beta$ phase.

To search for volume superconductivity in these $\beta$-phase crystals, we have looked for diamagnetic screening currents and for the Meissner effect with both ac as well as dc techniques. The ac technique consists of a tank circuit oscillating at $42 \mathrm{kHz}$ which is driven by a tunnel diode (GE BD-4) located in the helium bath of the cryostat. For maximum sensitivity, the oscillator coil is small $(0.8 \mathrm{~mm}$ inside diameter, $1.0 \mathrm{~mm}$ outside diameter, length $5 \mathrm{~mm}$ ) to accommodate the small crystal samples (typical dimensions
$0.5 \times 0.5 \times 0.2 \mathrm{~mm}^{3}$ ) and to yield a good filling factor. The dc technique consists of an inductance bridge with a superconducting quantum interference device (SQUID) null detector, as described before. ${ }^{11}$ The two inductance arms of the bridge, which contain the sample and a tin reference sample, respectively, can, at a low temperature of $0.1 \mathrm{~K}$, be loaded with supercurrent, creating measuring fields between 0.005 and $50 \mathrm{Oe}$. The temperature can then be raised towards $T_{c}$ and the decay of the SC screening currents can be observed by corresponding changes in the SQUID sensor current. The samples are actually mounted on an axis threading the two inductance coils of the bridge and can be rotated by means of a mechanism while they are cold. This dc technique has been employed before on other organic superconductors to detect even minute fractions of volume superconductivity (also in samples under pressure). ${ }^{12}$ The

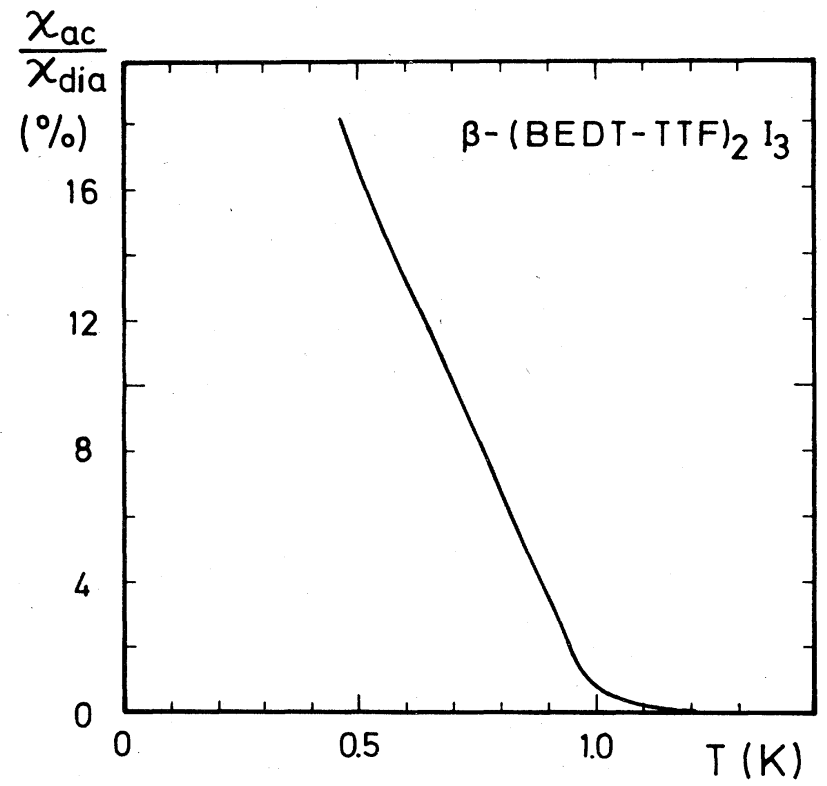

FIG. 1. ac susceptibility of $\beta$-(BEDT-TTF) ${ }_{2} I_{3}$ as a function of temperature in zero magnetic field. 


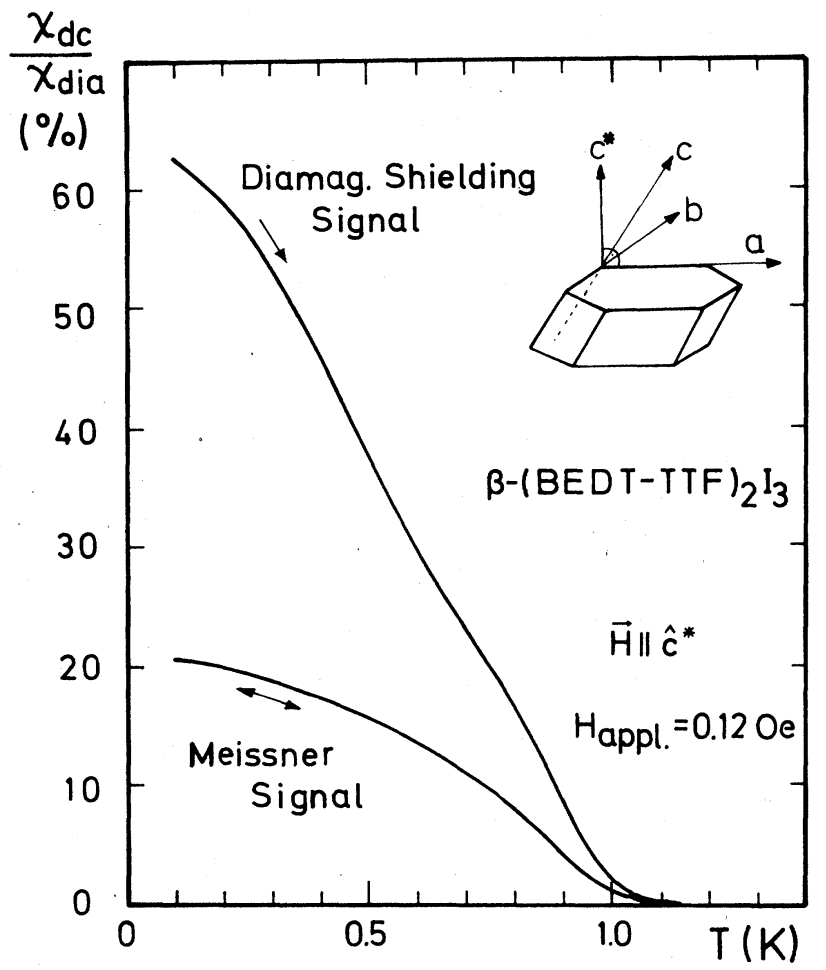

FIG. 2. Diamagnetic shielding and Meissner susceptibility vs temperature of $\beta$-(BEDT-TTF $)_{2} \mathrm{I}_{3}$ in a field applied perpendicular to the $a b$ plane. The insert relates the morphology of the crystal to its axes.

samples were always cooled with similar speeds, i.e., in about $6 \mathrm{~h}$ to $100 \mathrm{~K}$ and in $1.5 \mathrm{~h}$ to $4.2 \mathrm{~K}$. Figure 1 shows the result of an ac susceptibility measurement $(\nu=42 \mathrm{kHz}$, ac amplitude $=0.4 \mathrm{Oe}$ ) of three randomly oriented small crystals of $\beta$-(BEDT-TTF $)_{2} \mathrm{I}_{3}$. There is clear evidence of an onset of diamagnetic shielding currents below $1.05 \mathrm{~K}$. The signal, which still increases on cooling at $0.5 \mathrm{~K}$, is about $30 \%$ of that expected for a perfect superconductor and indicates an inhomogeneous $T_{c}$ distribution in the samples. The dc measurements were carried out on one crystal for three different field orientations. The relation between the morphology of the crystal and its triclinic axes, as reported from a structure analysis ${ }^{13}$ is indicated in the inset of Fig. 2. The conducting stacks of the BEDT-TTF molecules are reported to lie in the [110] direction, i.e., in the $a b$ plane, which is also the plane of highest conductivity and least conductivity anisotropy. The largest diamagnetic shielding and the largest Meissner signals (62\% and 18\%, respective-

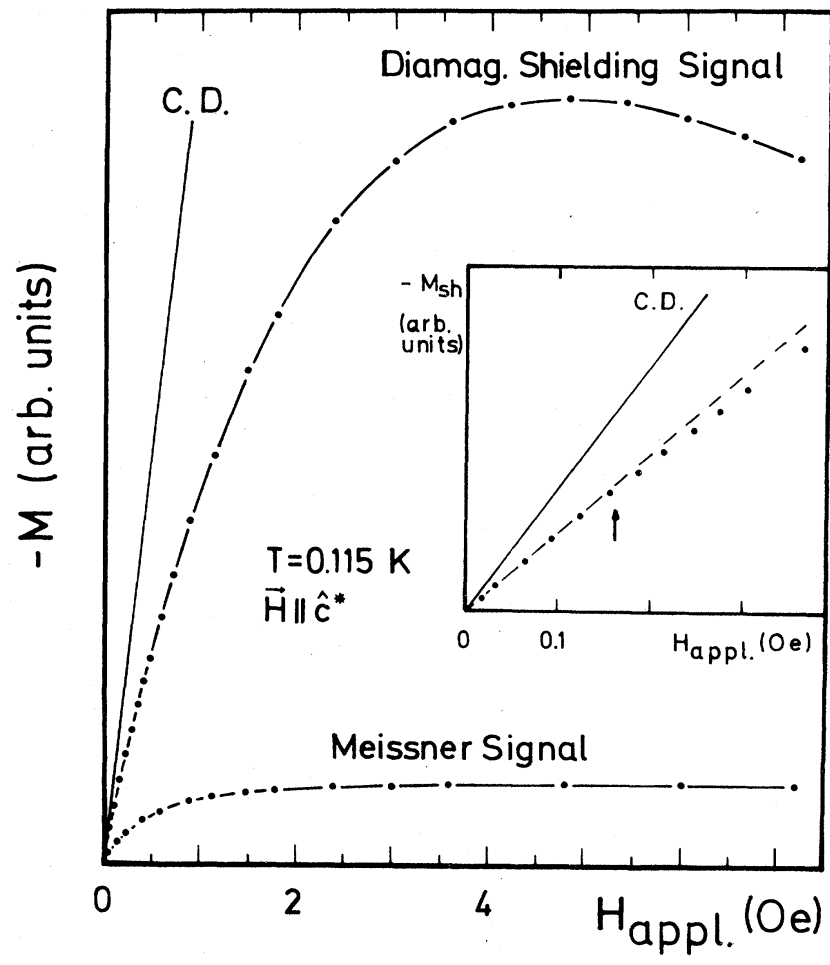

FIG. 3. Diamagnetic shielding and Meissner magnetization of $\beta$ (BEDT-TTF) ${ }_{2} \mathrm{I}_{3}$ vs field (applied perpendicular to the $a b$ plane) at $T=0.115 \mathrm{~K}$. The straight line (C.D.) would indicate complete diamagnetism, i.e., the shielding behavior of a perfect superconductor. The insert shows the enlarged low-field region of the diamagnetic shielding magnetization; the arrow indicates $H_{c 1}$ (see text).

ly, of that of a perfect SC at $T=0.115 \mathrm{~K}$ ) are observed when the field is oriented normal to this plane $\left(\mathbf{H} \| \hat{c}^{*}\right)$, i.e., when the supercurrents flow in this plane. This is shown in Fig. 2. Again, as in the ac measurement, it can be seen that the "diamagnetic" $T_{c}$ is $1.05 \mathrm{~K}$ (linearly extrapolated). From temperature sweeps in different fields, the diamagnetic magnetization curve can be constructed. This curve is shown in Fig. 3 for a temperature of $0.11 \mathrm{~K}$. It shows the typical type-III behavior observed in all organic superconductors so far. From the first deviation from linearity in low fields, an upper limit of $H_{c 1 c^{*}}$ of 0.36 Oe can be deduced (after correction for demagnetization effects).

When tilting the field into the $a b$ plane, we find smaller values of $H_{c 1}$ and, especially, smaller Meissner signals. Those results are summarized in Table $I$. The main reason for this is the anisotropy of the superconducting coherence

TABLE I. The values of the initial diamagnetic shielding susceptibilities $\left(\chi_{\mathrm{sh}}\right)$, the relative low-field Meissner magnetizations $\left(M_{\mathrm{Mei}} / M_{\mathrm{sh}}\right)$ and the lower critical fields $\left(H_{c 1}\right)$ for the different field orientations are given at $T=0.115 \mathrm{~K}$ and are corrected for demagnetization effects. The diamagnetic critical temperature is $1.03 \pm 0.02 \mathrm{~K}$

\begin{tabular}{cccc}
\hline $\begin{array}{c}\text { Field } \\
\text { orientation }\end{array}$ & $\begin{array}{c}\chi_{\text {sh }} \\
\text { (in } \% \text { of }-1 / 4 \pi)\end{array}$ & $\begin{array}{c}M_{\text {Mei }} / M_{\text {sh }} \\
(\%)\end{array}$ & $\begin{array}{c}H_{c 1} \\
(\mathrm{Oe})\end{array}$ \\
\hline $\mathbf{H}_{\|} \hat{\mathbf{a}}$ & 17.5 & 8.5 & $0.052 \pm 0.006$ \\
$\mathbf{H}_{1} \hat{\mathbf{a}}$ & 40.4 & $8.5 \pm 1$ & $0.087 \pm 0.014$ \\
$\mathbf{H}_{\perp}$ the $a b$ plane & $62 \pm 5$ & $29.5 \pm 3$ & $0.358 \pm 0.036$ \\
\hline \hline
\end{tabular}


length, which is expected to be largest in the $a b$ plane and smallest in the $c^{*}$ direction, i.e., normal to the layers of the cation stacks. With the field in the $a b$ plane, the magnetic vortices in the type-II state are thus smallest and are apparently more easily pinned by imperfections, leading to a small Meissner effect. Similar behavior was seen, e.g., in (TMTSF) ${ }_{2} \mathrm{CIO}_{4}$ (Ref. 12).

Our observations thus leave no doubt that $\beta$-(BEDTTTF $)_{2} I_{3}$ is an ambient pressure volume superconductor. When comparing the $\alpha$ and $\beta$ phases of this compound, we find that in the former, the cation molecules on neighboring stacks are tilted against one another, whereas in the latter they are not. The latter structure (the $\beta$ phase) thus actually resembles that of a Bechgaard salt. The question as to what causes the inhomogeneity in $T_{c}$, which leads to a resistance drop before the onset of diamagnetic screening currents, remains to be answered.

This work was supported by the Stiftung Volkswagenwerk in Hannover.
${ }^{1}$ E. B. Yagubskii, I. F. Shchegolev, V. N. Laukhin, P. A. Kononovich, M. B. Karatsovnik, A. V. Zvarykina, and L. J. Buravov, Pis'ma Zh. Eksp. Teor. Fiz. 39, 12 (1984) [JETP Lett. 39, 12 (1984)].

${ }^{2}$ S. S. P. Parkin, E. M. Engler, R. R. Schumaker, R. Lagier, V. Y. Lee, J. Voiron, K. Carneiro, J. C. Scott, and R. L. Greene, Phys. Rev. Lett. 50, 270 (1983).

${ }^{3}$ R. C. Lacoe, S. A. Wolf, P. M. Chaikin, F. Wudl, and E. AharonShalom, Phys. Rev. B 27, 1947 (1983).

${ }^{4}$ R. C. Lacoe, P. M. Chaikin, F. Wudl, and E. Aharon-Shalom, J. Phys. (Paris) Colloq. 44, C3-767 (1983).

${ }^{5}$ F. Gross, H. Schwenk, K. Andres, F. Wudl, S. D. Cox, and J. Brennan, Phys. Rev. B 30, 1282 (1984).

${ }^{6}$ K. Bender, K. Dietz, H. Endres, H. W. Helberg, I. Hennig, H. J. Keller, H. W. Schafer, and D. Schweitzer, Mol. Cryst. Liq. Cryst. 107, 45 (1984).

${ }^{7}$ K. Bender, I. Hennig, D. Schweitzer, K. Dietz, H. Endres, and H.
J. Keller, Mol. Cryst. Liq. Cryst. 108, 359 (1984).

${ }^{8}$ F. Gross, C. P. Heidmann, H. Schwenk, K. Andres, D. Schweitzer, and H. J. Keller, in Proceedings of the International Conference on the Physics and Chemistry of Low-Dimensional Synthetic Metals, Abano Terme, 1984 [Mol. Cryst. Liq. Cryst. (in press)].

${ }^{9}$ E. B. Yagubskii, I. F. Shchegolev, S. I. Pesotski, V. N. Laukhin, P. A. Kononovich, M. B. Karatsovnik, and A. V. Zvarykina, Pis'ma Zh. Eksp. Teor. Fiz. 39, 275 (1984) [JETP Lett. 39, 328 (1984)].

${ }^{10} \mathrm{R}$. P. Shibaeva, in Proceedings of the International Conference on the Physics and Chemistry Low-Dimensional Synthetic Metals, Abano Terme, 1984 [Mol. Cryst. Liq. Cryst. (in press)].

${ }^{11}$ K. Andres, F. Wudl, D. B. McWhan, G. A. Thomas, D. Nalewajek, and A. L. Stevens, Phys. Rev. Lett. 45, 1449 (1980).

${ }^{12} \mathrm{~K}$. Andres, H. Schwenk, and F. Wudl, Helv. Phys. Acta 55, 675 (1982).

${ }^{13} \mathrm{H}$. W. Helberg (private communication). 